\title{
THE CHOICE OF CAPACITY IN MIXED DUOPOLY UNDER DEMAND UNCERTAINTY*
}

\author{
by \\ YUANZHU LU \\ and \\ SOUGATA PODDAR \\ Department of Economics, National University of Singapore
}

\begin{abstract}
We analyze the capacity choice of firms under demand uncertainty in a mixed duopoly market consisting of one private firm and one public firm. We define a two-stage game where firms choose capacity in the first stage without knowing which state of Nature is going to be realized, and output in the second stage knowing which state is realized. We address the question of maintaining over and under capacity in the equilibrium as a strategic device; and show that both symmetric and asymmetric outcomes can be realized.
\end{abstract}

\section{INTRODUCTION}

The sequential choice of capacity and quantity by firms in a strategic environment has been carefully studied in the literature of industrial organization (see Spence, 1977; Dixit, 1980; Tirole, 1988, among many others). The issue of choosing over (excess) capacity or under capacity from a strategic point of view by the competing firms was always a matter of central debate. Various studies show the results can vary according to the modeling environment. Interestingly, most of the studies are performed when firms are pure profit maximizers. In recent years, study of mixed oligopolies, where a welfare-maximizing public firm interacts with profit-maximizing private firms, has become increasingly popular (see, for example, Cremer et al., 1989; DeFraja and Delbono, 1989; Anderson et al., 1997; White, 1997, among others). ${ }^{1}$ In this paper, we analyze a model of mixed duopoly with one public firm and one private firm. The public firm aims to maximize welfare (social surplus) and the private firm is the usual profit maximizer. We study a twostage competition between the two firms with capacity and quantity as strategic choice variables. Capacities are chosen simultaneously in the first stage and quantities are chosen simultaneously in the second stage. In addition to this, we also introduce an uncertain demand environment in the capacity

\footnotetext{
* Manuscript received 25.11.04; final version received 22.7.05.

${ }^{\dagger}$ We are thankful to one anonymous referee for valuable comments on an earlier draft. The remaining errors, if any, are ours.

${ }^{1}$ Mixed oligopolies are common in many countries. Oil industries, heavy manufacturing industries, telecommunications or the tourism industry are good examples of mixed oligopolies. 
choice stage. ${ }^{2}$ When firms install capacities, they do not know which state of demand (high, medium or low) will be realized in future. They choose respective quantities after uncertainty is resolved. In this set-up, we address the question of strategically choosing excess or under capacity by the competing firms in the equilibrium. We find that if the realized demand is high or low, the outcome of the game is symmetric between the firms in terms of choosing excess or under capacity; whereas if the realized demand is medium, the outcome is asymmetric, and, in particular, the public firm will end up choosing under capacity and the private firm will end up with excess capacity.

In this framework, the competing firms not only provide best replies against the capacity and output strategies chosen by the rival firm but also adjust their capacities in view of meeting output demand levels varying across the states of Nature. Thus, in this random environment, firms play a game simultaneously against Nature and against a rival firm, where the rival firm has a different objective. The resulting equilibrium outcomes are the interaction of the two effects influencing at the same time firms' behavior. ${ }^{3}$

\section{Model}

We consider a mixed duopoly market where a profit-maximizing private firm, firm $a$, and a social-welfare-maximizing public firm, firm $b$, are operating in a homogeneous good market. Social welfare (surplus) is defined as the sum of consumer surplus and both firms' profits.

We specify the cost function as

$$
C_{i}\left(q_{i}, x_{i}\right)=m_{i} q_{i}+\left(q_{i}-x_{i}\right)^{2}
$$

where $q_{i}$ and $x_{i}$ are the production quantity and capacity of firm $i(=a, b){ }^{4}$ We assume $m_{a}<m_{b}$; i.e. firm $a$ can produce more efficiently than firm $b$ at the efficient production-capacity level. ${ }^{5}$ Under this U-shaped cost function, the long-run average cost is minimized when quantity equals production capacity, i.e. $q_{i}=x_{i}$.

We assume that there are $n$ states of Nature and the demand in state $i$ is given by

$$
p_{i}(Q)=a_{i}-Q=a_{i}-\left(q_{a}+q_{b}\right) \quad i=1, \ldots, n
$$

${ }^{2}$ Recently, Nishimori and Ogawa (2004) analyzed the sequential choice of capacity and quantity in a mixed duopoly market when the demand is deterministic. The game with endogenous timing of choosing quantities in a mixed oligopoly was studied by Pal (1998).

${ }^{3}$ For some studies with demand uncertainty in a pure oligopoly framework (i.e. only profitmaximizing firms), see Perrakis and Warskett (1983), Gabszewicz and Poddar (1997) and Maskin (1999).

${ }^{4}$ The same cost structure is used in Horiba and Tsutsui (2000), Nishimori and Ogawa (2004) and elsewhere.

${ }^{5}$ Note that $m_{a} \geq m_{b}$ will yield zero profit for the private firm. Other technical conditions needed to guarantee positive equilibrium outputs and prices in the subsequent analysis are given in the Appendix. 
and

$$
a_{i}<a_{i+1} \quad i=1, \ldots, n-1
$$

where $p_{i}$ is market price, $Q$ is total output and $q_{a}$ and $q_{b}$ denote the output of firm $a$ and firm $b$, respectively.

We study a two-stage game where firms choose capacity in the first stage without knowing which state of Nature is going to be realized, and output in the second stage knowing which state is realized. We assume the existence of an objective probability density $\rho_{i}, i=1, \ldots, n$, over the states of Nature, $\sum_{i=1}^{n} \rho_{i}=1$. Firms are assumed to be risk neutral.

\section{The Subgame Perfect Equilibrium}

We look for subgame perfect Nash equilibrium.

Assume that firms have chosen capacities $x_{a}$ and $x_{b}$ in the first stage. We consider the second-stage game. The second-stage payoffs of firms $a$ and $b$ in state $i$ are given by

$$
\pi_{a}^{i}=\left(a_{i}-q_{a}^{i}-q_{b}^{i}\right) q_{a}^{i}-m_{a} q_{a}^{i}-\left(q_{a}^{i}-x_{a}\right)^{2}
$$

and

$$
\mathrm{SS}_{b}^{i}=a_{i}\left(q_{a}^{i}+q_{b}^{i}\right)-\frac{\left(q_{a}^{i}+q_{b}^{i}\right)^{2}}{2}-m_{a} q_{a}^{i}-m_{b} q_{b}^{i}-\left(q_{a}^{i}-x_{a}\right)^{2}-\left(q_{b}^{i}-x_{b}\right)^{2}
$$

Given their production capacities, the maximization problem of each firm yields

$$
\begin{aligned}
& q_{a}^{i}=\frac{a_{i}-m_{a}+2 x_{a}-q_{b}^{i}}{4} \\
& q_{b}^{i}=\frac{a_{i}-m_{b}+2 x_{b}-q_{a}^{i}}{3}
\end{aligned}
$$

By solving (5) and (6), we obtain the output levels as

$$
\begin{aligned}
q_{a}^{i} & =\frac{2 a_{i}-3 m_{a}+m_{b}+6 x_{a}-2 x_{b}}{11} \\
q_{b}^{i} & =\frac{3 a_{i}+m_{a}-4 m_{b}-2 x_{a}+8 x_{b}}{11}
\end{aligned}
$$

Next, we consider the first-stage game. Since we assume both firms are risk neutral, they choose capacity to maximize expected payoff without knowing which state is going to be realized. When they choose the capacity scale, they know that their decision affects their output decision in the second stage. Hence, we can formulate the maximization problem of the private firm as follows: 


$$
\max _{x_{a}} E\left[\pi_{a}\right]=\sum_{i=1}^{n} \rho_{i}\left[\left(a_{i}-q_{a}^{i}-q_{b}^{i}\right) q_{a}^{i}-m_{a} q_{a}^{i}-\left(q_{a}^{i}-x_{a}\right)^{2}\right]
$$

subject to (7) and (8)

The first-order condition is

$$
x_{a}=\frac{12}{49} 2 \sum_{i=1}^{n} \rho_{i} a_{i}-3 m_{a}+m_{b}-2 x_{b}
$$

Similarly, the public firm's maximization problem can be formulated as

$$
\begin{aligned}
\max _{x_{b}} E\left[\operatorname{SS}_{b}^{i}\right]= & \sum_{i=1}^{n} \rho_{i}\left[a_{i}\left(q_{a}^{i}+q_{b}^{i}\right)-\frac{\left(q_{a}^{i}+q_{b}^{i}\right)^{2}}{2}\right. \\
& \left.-m_{a} q_{a}^{i}-m_{b} q_{b}^{i}-\left(q_{a}^{i}-x_{a}\right)^{2}-\left(q_{b}^{i}-x_{b}\right)^{2}\right]
\end{aligned}
$$

subject to (7) and (8)

The first-order condition is

$$
x_{b}=\sum_{i=1}^{n} \rho_{i} a_{i}-m_{b}-\frac{14\left(m_{b}-m_{a}+2 x_{a}\right)}{31}
$$

From (7)-(10), we can get both firms' production quantity and capacity level:

$$
\begin{aligned}
& x_{a}=\frac{12\left(m_{b}-m_{a}\right)}{7} \\
& x_{b}=\sum_{j=1}^{n} \rho_{j} a_{j}-3 m_{b}+2 m_{a} \\
& q_{a}^{i}=\frac{11\left(m_{b}-m_{a}\right)}{7}+\frac{2\left(a_{i}-\sum_{j=1}^{n} \rho_{j} a_{j}\right)}{11} \\
& q_{b}^{i}=a_{i}-3 m_{b}+2 m_{a}+\frac{m_{b}-m_{a}}{7}+\frac{8\left(\sum_{j=1}^{n} \rho_{j} a_{j}-a_{i}\right)}{11}
\end{aligned}
$$

Now, comparing (13) with (11) and (14) with (12) we get Table 1. This leads us to our main result.

Proposition: In the two-stage game in which both the public firm and the private firm choose their capacities simultaneously without knowing the true state of demand and then both choose quantities simultaneously after the demand is realized, we get two symmetric and one asymmetric outcome in terms of equilibrium capacity choice, depending on the strength of the realized demand. 
TABLE 1

Choice of Capacity under Different Realized Demand

\begin{tabular}{lcc}
\hline Realized demand & Public firm & Private firm \\
\hline$a_{i} \leq \sum_{j=1}^{n} \rho_{j} a_{j}-11\left(m_{b}-m_{a}\right) / 21$ & Excess capacity & $\begin{array}{c}\text { Excess capacity } \\
\left(x_{a}>q_{a}\right)\end{array}$ \\
$\sum_{j=1}^{n} \rho_{j} a_{j}-11\left(m_{b}-m_{a}\right) / 21<a_{i}<\sum_{j=1}^{n} \rho_{j} a_{j}+11\left(m_{b}-m_{a}\right) / 14$ & Under capacity & $\begin{array}{c}\text { Excess capacity } \\
\left(x_{b}>q_{a}\right)\end{array}$ \\
$a_{i} \geq \sum_{j=1}^{n} \rho_{j} a_{j}+11\left(m_{b}-m_{a}\right) / 14$ & Under capacity & Under capacity \\
& $\left(x_{b}<q_{b}\right)$ & $\left(x_{a}<q_{a}\right)$ \\
\hline
\end{tabular}

(i) Symmetric outcome: (a) when the realized demand is low, both firms carry idle or excess capacity; (b) when the realized demand is high, both firms' quantities exceed their capacities.

(ii) Asymmetric outcome: when the realized demand is medium, the public firm's quantity exceeds its capacity and the private firm carries idle capacity.

We believe that, when the demand is too high or low, the strategic effect is overshadowed by the strong uncertainty effect resulting in a symmetric outcome, despite the difference in the respective objective functions, whereas when the demand is medium, it is more like the average demand, and there the strategic effect overshadows the (relatively weak) uncertainty effect, resulting in an asymmetric outcome where the public firm chooses under capacity while the private firm chooses over (excess) capacity. Since the private firm is more efficient, the public firm tries to make the private firm produce more while it produces less. Hence, the public firm reduces its own capacity so that the private firm can produce more. Meanwhile, enlarging the production share in the market is desirable for the private firm. Thus, the private firm chooses over capacity while the public firm chooses under capacity as a strategic device.

\section{Concluding Remarks}

Here, we would like to compare the outcomes in the mixed duopoly case with the outcome in the purely private duopoly case.

Two private firms playing such a capacity-then-quantity game under deterministic demand is exactly the benchmark case in Horiba and Tsutsui (2000). ${ }^{6}$ The outcome is that both firms choose excess capacity. Specifically, $x_{a}=x_{b}=16(a-m) / 43$ and $q_{a}=q_{b}=15(a-m) / 43$.

${ }^{6}$ In Horiba and Tsutsui (2000), the demand function is $p(Q)=\alpha-b Q$ and the cost function is $C\left(q_{i}, x_{i}\right)=m q_{i}+c\left(q_{i}-x_{i}\right)^{2}$. When we use the results on p. 211, especially equations (3) and (4a), we need to note the difference in notation, demand and cost function. That is, ' $a$ ' (= $\alpha-m$ ) in equations (3) and (4a) should be replaced by ' $a-m$ ', $b=1$, and $k \equiv b / c=1$. 
TABLE 2

Choice of CAPacity under Different

Realized Demand

\begin{tabular}{lc}
\hline Realized demand & Choice of capacity \\
\hline$a_{i} \leq\left(48 \sum_{j=1}^{n} \rho_{j} a_{j}-5 m\right) / 43$ & Excess capacity \\
$a_{i} \geq\left(48 \sum_{j=1}^{n} \rho_{j} a_{j}-5 m\right) / 43$ & Under capacity \\
\hline
\end{tabular}

If two private firms play such a game under demand uncertainty, then we can show that both firms may choose under capacity or excess capacity depending on the strength of the realized demand. When the realized demand is high enough, both firms' quantities exceed their capacities; otherwise, both firms carry idle capacity. And the results are as follows: $x_{a}=x_{b}=$ $16\left(\sum_{i=1}^{n} \rho_{i} a_{i}-m\right) / 43$ and $q_{a}=q_{b}=\left(a_{i}-m\right) / 5+32\left(\sum_{i=1}^{n} \rho_{i} a_{i}-m\right) / 215$. Table 2 presents firms' choice of capacity under different realized demand.

Thus, in these two situations, we always get a symmetric outcome. That is, regardless of deterministic demand or uncertain demand, the outcome is symmetric between the firms in terms of excess or under capacity in a pure oligopoly (i.e. when firms are only profit maximizers). However, both symmetric and asymmetric outcomes may arise in a mixed oligopoly.

\section{ApPendix: Restrictions on Parameters}

We need to impose some restrictions on parameters to make sure firms' capacities, quantities and market prices in equilibrium are positive.

(1) $x_{a}=12\left(m_{b}-m_{a}\right) / 7$ is always positive.

(2) $x_{b}=\sum_{j=1}^{n} \rho_{j} a_{j}-3 m_{b}+2 m_{a}$ is positive when $\sum_{j=1}^{n} \rho_{j} a_{j}>3 m_{b}-2 m_{a}$.

(3) Since $q_{a}^{i}=11\left(m_{b}-m_{a}\right) / 7+2\left(a_{i}-\sum_{j=1}^{n} \rho_{j} a_{j}\right) / 11$ and $q_{b}^{i}=a_{i}-3 m_{b}+2 m_{a}+\left(m_{b}-m_{a}\right) / 7$ $+8\left(\sum_{j=1}^{n} \rho_{j} a_{j}-a_{i}\right) / 11=3 a_{i} / 11+8 \sum_{j=1}^{n} \rho_{j} a_{j} / 11-20 m_{b} / 7+13 m_{a} / 7$ are increasing in $a_{i}$, we only require $q_{a}^{1}>0$ and $q_{b}^{1}>0$. The condition is $a_{1}>\max \left\{\sum_{j=1}^{n} \rho_{j} a_{j}-121\left(m_{b}-\right.\right.$ $\left.\left.m_{a}\right) / 14,220 m_{b} / 21-143 m_{a} / 21-8 \sum_{j=1}^{n} \rho_{j} a_{j} / 3\right\}$.

(4) Since $p_{i}(Q)=9 m_{b} / 7-2 m_{a} / 7+6\left(a_{i}-\sum_{j=1}^{n} \rho_{j} a_{j}\right) / 11$ is also increasing in $a_{i}$, we only require $p_{1}(Q)=9 m_{b} / 7-2 m_{a} / 7+6\left(a_{1}-\sum_{j=1}^{n} \rho_{j} a_{j}\right) / 11>0$. The condition is $a_{1}>\sum_{j=1}^{n} \rho_{j} a_{j}$ $-33 m_{b} / 14+11 m_{a} / 21$.

Hence, we need to impose the following restrictions on parameters to make equilibrium capacities, quantities and prices positive:

$$
\sum_{j=1}^{n} \rho_{j} a_{j}>3 m_{b}-2 m_{a}
$$


and

$$
\begin{aligned}
a_{1}>\max & \left\{\sum_{j=1}^{n} \rho_{j} a_{j}-\frac{121\left(m_{b}-m_{a}\right)}{14}, \frac{220 m_{b}}{21}-\frac{143 m_{a}}{21}\right. \\
& \left.-\frac{8}{3} \sum_{j=1}^{n} \rho_{j} a_{j}, \sum_{j=1}^{n} \rho_{j} a_{j}-\frac{33 m_{b}}{14}+\frac{11 m_{a}}{21}\right\}
\end{aligned}
$$

\section{REFERENCES}

Anderson, S., de Palma, A. and Thisse, J. (1997). 'Privatization and Efficiency in a Differentiated Industry', European Economic Review, Vol. 41, pp. 1635-1654.

Cremer, H., Marchand, M. and Thisse, J. (1989). 'The Public Firm as an Instrument for Regulating an Oligopolistic Market', Oxford Economic Papers, Vol. 41, pp. 283-301.

DeFraja, G. and Delbono, F. (1989). 'Alternative Strategies of a Public Enterprise in Oligopoly', Oxford Economic Papers, Vol. 41, pp. 302-311.

Dixit, A. (1980). 'The Role of Investment in Entry Deterrence', Economic Journal, Vol. 90, pp. 95-106.

Gabszewicz, J. and Poddar, S. (1997). 'Demand Fluctuations and Capacity Utilization under Duopoly', Economic Theory, Vol. 10, pp. 131-146.

Horiba, Y. and Tsutsui, S. (2000). 'International Duopoly, Tariff Policies and the Case of Free Trade', Japanese Economic Review, Vol. 51, pp. 207-220.

Maskin, E. (1999). 'Uncertainty and Entry Deterrence', Economic Theory, Vol. 14, pp. 429-437.

Nishimori, A. and Ogawa, H. (2004). 'Do Firms Always Choose Excess Capacity?', Economics Bulletin, Vol. 12, pp. 1-7.

Pal, D. (1998). 'Endogenous Timing in a Mixed Oligopoly', Economics Letters, Vol. 61, pp. $181-185$.

Perrakis, S. and Warskett, G. (1983). 'Capacity and Entry Deterrence under Demand Uncertainty', Review of Economics Studies, Vol. 50, pp. 495-511.

Spence, A. M. (1977). 'Entry, Capacity, Investment and Oligopolistic Pricing', Bell Journal of Economics, Vol. 8, pp. 534-544.

Tirole, J. (1988). The Theory of Industrial Organization, Cambridge, MA, MIT Press. White, M. (1997). 'Mixed Oligopoly, Privatization and Subsidization', Economics Letters, Vol. 53, pp. 189-195. 